\title{
Undifferentiated Pleomorphic Sarcoma With Hyaline Globules (Thanatosomes)
}

\author{
Mitsuhiro Tachibana ${ }^{1}$, Kei Tsukamoto ${ }^{2}$, Mitsuru Takahashi ${ }^{3}$, Yutaka Tsutsumi ${ }^{4,1}$ \\ 1. Department of Diagnostic Pathology, Shimada City General Medical Center, Shimada, JPN 2. Department of \\ Diagnostic Radiology, Shimada City General Medical Center, Shimada, JPN 3. Department of Orthopaedic Surgery, \\ Shizuoka Cancer Center Hospital and Research Institute, Suntou, JPN 4. Diagnostic Pathology Clinic, Pathos Tsutsumi, \\ Inazawa, JPN
}

Corresponding author: Mitsuhiro Tachibana,0206mtachi@gmail.com

\section{Abstract}

Hyaline globules (HGs) or thanatosomes belong to a well-defined microscopic phenomenon common to any cell type, representing eosinophilic and round-shaped intracytoplasmic inclusions as a result of altered cellular metabolism. We experienced a case of undifferentiated pleomorphic sarcoma (UPS) of the left thigh, immunoreactive diffusely for CD 99 and $\mathrm{p} 16^{\text {INK4a }}$ and focally for alpha-smooth muscle actin. HGs were multifocally clustered in the cytoplasm of the tumor cells. An ultrastructural study using a formalin-fixed, paraffin-embedded block was performed to visualize HGs in the UPS cells. Light microscopically, multifocally clustered HGs were PAS-positive with diastase-resistance and fuchsinophilic in Masson's trichrome staining. HGs were immunoreactive for cleaved caspase-3, but negative for ubiquitin. Ultrastructurally, apoptotic tumor cells contained clusters of small-sized electron-dense globules. Granular material was often deposited in the globule matrix. The formation of the HGs is supposedly related to an apoptotic process of the tumor cells. Though a nonspecific and minor microscopic finding, HGs in soft tissue sarcomas may represent a useful histologic marker of enhanced cell turnover and/or ischemic injury. This is the third report describing HGs in UPS.

Review began 05/30/2021 Review ended 06/06/2021 Published 06/21/2021

\section{(c) Copyright 2021}

Tachibana et al. This is an open access article distributed under the terms of the Creative Commons Attribution License CC-BY 4.0., which permits unrestricted use, distribution, and reproduction in any medium, provided the original author and source are credited.
Categories: Pathology, Orthopedics, Other

Keywords: hyaline globules, thanatosomes, undifferentiated pleomorphic sarcoma

\section{Introduction}

Hyaline globules (HGs), also termed as thanatosomes, have been identified in various types of cells and tissues in the normal, subnormal, nonneoplastic and neoplastic conditions [1]. HGs represent a welldescribed microscopic phenomenon that may serve as a hallmark of accelerated cell turnover and apoptosis $[1,2]$. The term "thanatosomes" derived from the Greek words "thanatos" meaning the death and "soma" meaning the body. The presence of HGs or thanatosomes has been reported in such soft tissue malignancies as Kaposi's sarcoma, cartilaginous neoplasms, malignant peripheral nerve sheath tumor and undifferentiated embryonal sarcoma of liver [3-6]. Undifferentiated pleomorphic sarcoma (UPS), previously known as malignant fibrous histiocytoma [7], may also accompany HGs in the cytoplasm [8,9]. We report herein a case of UPS of the left thigh, rich in HGs. Immunohistochemical and ultrastructural evaluation of HGs is described.

\section{Case Presentation}

\section{Clinical Summary}

A Japanese woman nonsmoker aged in her 60's was referred to Shimada Municipal Hospital, Shimada, Japan. She noticed an asymptomatic lump of her left thigh. Detailed radiological examinations, including magnetic resonance imaging, diagnosed the lesion as malignant soft tissue tumor (Figure 1A, 1B). A T2weighted heterogeneous intensity mass was seen in the skeletal muscle of the left thigh. Neither lymphadenopathy nor metastasis was noted. Incisional biopsy of the lesion was performed. 

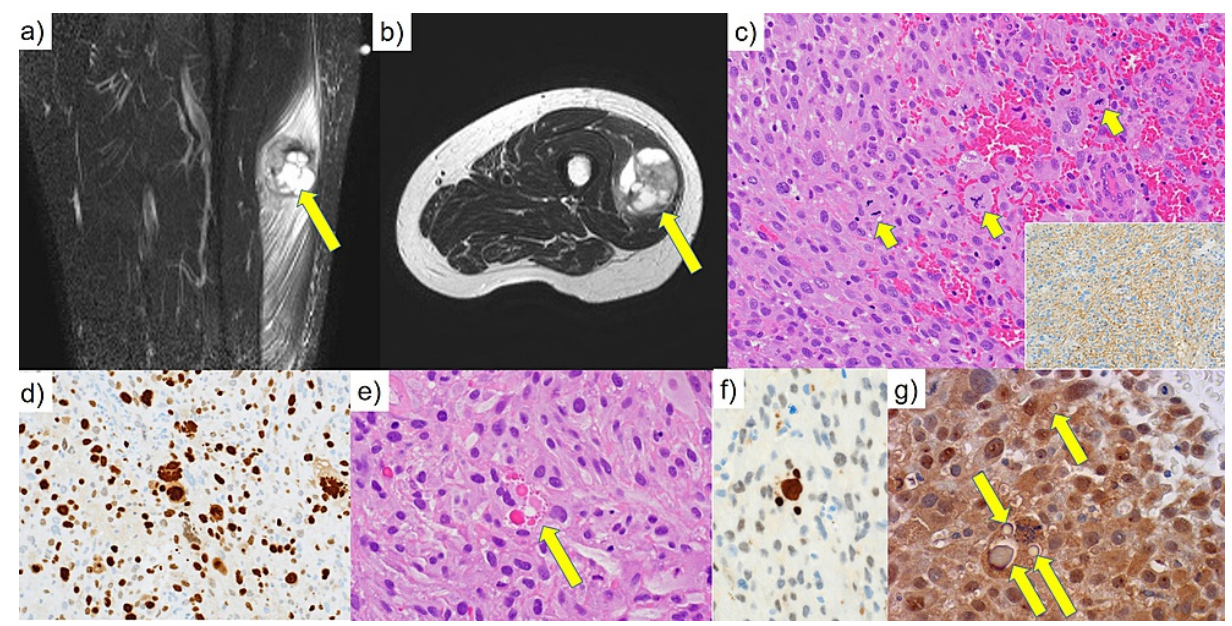

FIGURE 1: Radiological and light microscopic findings of UPS with HGs.

$(\mathrm{A}, \mathrm{B})$ On magnetic resonance imaging, a T2-weighted heterogeneous intensity mass, as indicated by arrows, is seen in the skeletal muscle of the left thigh [(A) coronal section, (B) transverse section]. (C) The tumor is composed of pleomorphic spindled, stellate or epithelioid cells frequently mitotic figures (arrows) (H\&E). Bizarre nuclei are scattered. The tumor cells are immunoreactive diffusely for CD99 (MIC-2) (inset). (D)Immunostaining for Ki-67. (E) Intracytoplasmic hyaline globules (HGs) (thanatosomes) are clustered (arrow) (H\&E). Immunohistochemistry, the HGs were positive for cleaved caspase-3 (F), but negative for ubiquitin (G).

\section{Light microscopic, histochemical and immunohistochemical analysis of the biopsy material}

Light microscopically, the mass was composed of pleomorphic spindled, stellate or epithelioid cells (Figure 1C), frequently accompanying foci of coagulation necrosis. Bizarre nuclei were scattered. The cytoplasm was relatively plump and eosinophilic. Mitoses, including atypical ones, were easily observed (48 in 10 highpowered fields; Figure 1C). The tumor cells were immunoreactive diffusely for CD99 (MIC-2) (Figure 1C, inset) and p16-INK4A, and moderately for a-smooth muscle actin. Negative markers included S-100 protein, desmin, heavy-caldesmon, cytokeratins, epithelial membrane antigen, p53, cyclin-dependent kinase-4 (CDK4), murine double minute-2 (MDM2), and CD34. Ki-67 labeling index was 56.9-95.8\%, with the mean $62.9 \%$ (Figure $1 D$ ). Some tumor cells possessed HGs of varying sizes in the cytoplasm (Figure $1 E$ ). Their sizes ranged from 1.0 to 18.6 micrometers, with the mean value at 5.4 and median at 3.2. HGs revealed periodic acid-Schiff (PAS) reactivity with amylase resistance and fuchsinophilia in Masson's trichrome stain. The HGs were positive for cleaved caspase-3 (Figure $1 F$ ), but negative for ubiquitin (Figure $1 \mathrm{G}$ ). The final diagnosis was UPS, accompanying HGs (thanatosomes), grade 3, according to the Federation Nationale des Centres de Lutte le Cancer.

\section{Ultrastructural studies}

A paraffin block of the biopsy specimen was processed for ultrastructural observation, as reported previously $[10,11]$. The specimen was routinely fixed in $0.01 \mathrm{M}$ phosphate-buffered $10 \%$ formalin, pH 7.4 (Kanto Chemical, Tokyo) and embedded in paraffin (Parabett 60 GR, Muto Pure Chemicals, Tokyo). The area with tumor cells containing clustered HGs was dug out of the paraffin block as $1 \mathrm{~mm}$-sized cubes. After deparaffinization overnight, and the tissue block was soaked through graded series of alcohol. The rehydrated block was re-fixed at $4 \mathrm{C}$ overnight in $2.5 \%$ glutaraldehyde (Yuai Kasei, Amagasaki, Japan) buffered with $0.1 \mathrm{M}$ sodium cacodylate at $\mathrm{pH}$ 7.4, osmified for 2 hours with sodium cacodylate-buffered $1 \%$ osmium tetraoxide (Nisshin EM, Tokyo), embedded in epoxy resin (Epon 812, Okenshoji, Tokyo), and polymerized overnight in a 70C oven. Ultrathin sections were prepared using a Diatome diamond (JEOL Japan, Tokyo) at $80 \mathrm{~nm}$ thickness, and stained with uranyl acetate (Ieda Chemicals, Tokyo) and lead citrate (Sigma Aldrich Japan, Tokyo). Images were photographed on a JEOL JEM1400 Flash Electron microscope (JEOL Japan) equipped with an EM-14661FLASH high-sensitivity digital complementary metal-oxidesemiconductor camera.

Fine structural preservation was satisfactory, though not ideal. The spindled or polygonal tumor cells ultrastructurally possessed round or oval nuclei with increased euchromatin and prominent nucleoli (Figure $2 A)$. Fine filaments were occasionally clustered in the cytoplasm. Lipid droplets were not observed. Lysosomal granules represented autophagic vacuoles. Electron-dense, round-shaped globules (HGs) were formed in the tumor cells (Figure 2B,2C). The size of the intracytoplasmic globules ranged from 1.0 to 18.6 micrometers, with the mean value at 5.4 micrometers and median at 3.2 micrometers. The globules were not necessarily homogenous, and granular material was deposited in the matrix of the globules (Figure $2 B, 2 C$ ). The globules were not surrounded by rough endoplasmic reticulum. The 


\section{Cureus}

ultrastructural features of the globules were not typical of HGs caused by the secretory disturbance occasionally seen in epithelial cells, plasma cells or adrenomedullary cells. In combination with positive cleaved caspase-3 immunoreactivity, the HGs are supposedly related to an apoptotic process of the tumor cells, representing as genuine thanatosomes (death bodies).

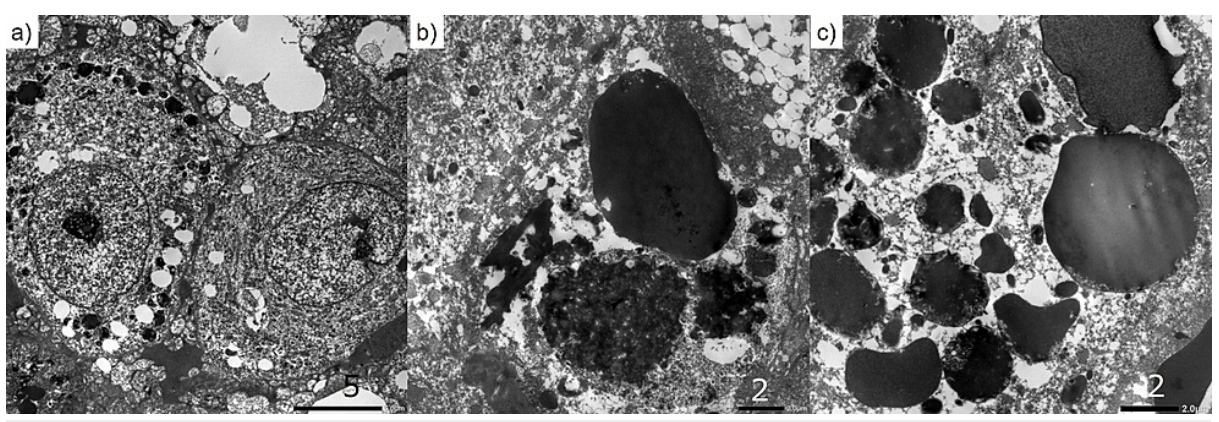

FIGURE 2: Ultrastructural findings of UPS with HGs.

(a) The polygonal-shaped neoplastic cells possess round or oval nuclei with increased euchromatin and prominent nucleoli. (b,c) HGs are formed in some tumor cells. The globules are not homogenous, and granular material is deposited in the matrix of the globules. The globules are not surrounded by rough endoplasmic reticulum. Some apoptotic tumor cells contain clusters of smaller-sized electron-dense globules, ranging from 1.0 to 18.6 micrometers. The maximal size of HGs reaches 18.6 micrometers. [bars = 5 micrometers (a), 2 micrometers (b), 2 micrometers (c)]

\section{Follow-up information}

Neoadjuvant chemotherapy [Adriamycin+Ifosfamide (AI)] was performed for three courses in the Shizuoka Cancer Center Hospital, Nagaizumi, Shizuoka, Japan. Thereafter, the left thigh tumor was widely removed. The surgical specimen showed complete coagulation necrosis of the $65 \mathrm{~mm}$-sized demarcated tumor and microscopically contained no viable tumor cells. Adjuvant chemotherapy (AI for two courses) was added. Neither recurrence nor metastasis was noted for five years.

\section{Discussion}

The diagnosis of UPS was primarily made by anaplastic and pleomorphic histopathological features with high Ki-67 labeling index. The sarcoma cells were immunoreactive diffusely for CD99 and focally for alphasmooth muscle actin. CD99 expression and smooth muscle differentiation in UPS have been reported $[12,13]$. CDK4 and MDM2, intranuclear markers of high-grade liposarcoma [14], were negative. Lipid droplets were scarcely noted ultrastructurally. In contrast, $\mathrm{p} 16^{\mathrm{INK} 4 \mathrm{a}}$, a known immunohistochemical marker of senescence [15] and favorable prognostic marker of osteosarcoma [16] was observed, but no osteoid formation was noted in the tumor of the present case.

HGs or thanatosomes have been described in both neoplastic and nonneoplastic diseases in varied tissues and organs $[1,2,17-19]$. In hematoxylin and eosin-stained preparations, HGs typically appear as intracytoplasmic eosinophilic, rounded structures of varying size. HGs stain magenta with PAS reaction showing amylase-resistance, and appear fuchsinophilic in Masson's trichrome preparation. Ultrastructurally, the HGs consisted of large osmiophilic inclusions. Papadimiriou, et al. described that the HGs ultrastructurally appeared as phagosomes/secondary lysosomes or areas of cytoplasmic condensation surrounded by rough endoplasmic reticulum whorls [1]. Double stains for apoptotic markers and plasma proteins confirmed the increased plasma membrane permeability to allow accumulation of proteins in the apoptotic cells to form HGs [1].

Cleaved caspase-3, representing an activated form of caspase-3, is an excellent and reproducible immunohistochemical marker for apoptosis [20,21]. The biochemical pathways of apoptosis are controlled by caspases (cysteine aspartate-specific proteases), which cleave and activate a variety of intracellular proteins $[22,23]$. Cleaved caspase 3 functions as a kind of control tower of apoptosis: it cleaves poly (ADPribose) polymerase (PARP), cytokeratin 18, vimentin, actin and other intracytoplasmic proteins. Cleaved caspase 3 has been applied to detecting apoptotic neoplastic cells in paraffin sections [24-26].

In contrast, ubiquitin is ubiquitously expressed in all types of cells (hence the term ubiquitin), playing an important role in removing abnormal proteins from the cell. The ubiquitination functions as posttranslational modification to control multiple steps in autophagy, a major lysosome-mediated intracellular degradation pathway [27,28]. Amorphous cytoplasmic inclusions composed of aggregated ubiquitinated intermediate filament proteins are seen in a variety of metabolic disorders such as alcoholic hepatitis and neurodegenerative diseases [29]. 
In the present analysis, the HGs in UPS were immunohistochemically positive for cleaved caspase-3, but negative for ubiquitin. Ultrastructural appearance was also compatible with the apoptosis-related process. We concluded that the HGs were formed in relation to the apoptotic process in the sarcoma cells, as illustrated in Figure 3.
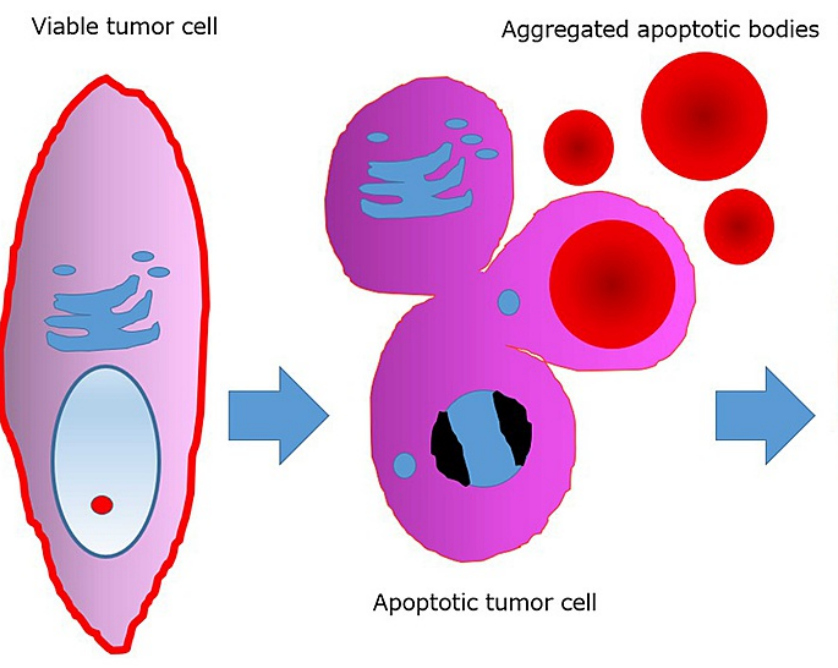

Engulfment by a nearby cell

\section{FIGURE 3: A model for the formation of HGs in the present neoplasm.}

An intact tumor cell responds to a variety of injurious stimuli toward apoptosis. The apoptotic bodies are engulfed by a neighboring viable cell.

There are two mechanisms for the formation of HGs. One is the above-mentioned apoptosis/ischemiarelated type, and another is caused by the secretory disturbance. Apoptotic nature has been suggested in HGs seen in the benign and neoplastic gastrointestinal epithelial cells [2]. Hyaline droplets (or HGs) formed in proximal renal tubules were often related to acute tubular necrosis, and even when tubular necrosis was not evident, the proximal tubular epithelial cells containing hyaline droplets ultrastructurally showed degenerated microvilli and decreased basal interdigitations [30]. In contrast, HGs seen in plasma cells (socalled Russell bodies) [31] and HGs formed in the adrenomedullary pheochromocytes [32] typically represent the disturbance in secretory activity of the cell. del Rosario, et al. [4] reported that intracytoplasmic eosinophilic HGs in cartilaginous neoplasms were suspicious for the secretory products of probable glycoprotein nature. HG-like structures were also described in undifferentiated sarcoma cells of malignant müllerian mixed tumor of the fallopian tube. The structures displayed electron-dense membrane-bound structures that were consistent with lysosomes [33]. We recently described two cases of intraductal papillary mucinous neoplasm of the pancreas accompanying HGs of secretory disturbance type [34].

\section{Conclusions}

To the best of our knowledge, there have been only two cases of UPS accompanying HGs, and the present study represents the first ultrastructural analysis of HGs in UPS. Further accumulation of cases is needed to clarify the incidence and clinicopathological significance of HGs in this type of high-grade sarcoma. The availability of routinely processed paraffin blocks for ultrastructural analysis should also be emphasized.

\section{Additional Information}

\section{Disclosures}

Human subjects: Consent was obtained or waived by all participants in this study. the Ethics Committee for Clinical Research of Shimada Municipal Hospital, Shimada, Shizuoka, Japan issued approval R02-16. All the procedures were in accordance with the ethical standards of the responsible institutional committee on human experimentation and with the Helsinki Declaration of 1964 and later versions. The patient gave a written informed consent to publication as a case report. . Conflicts of interest: In compliance with the ICMJE uniform disclosure form, all authors declare the following: Payment/services info: All authors have declared that no financial support was received from any organization for the submitted work. Financial relationships: All authors have declared that they have no financial relationships at present or within the previous three years with any organizations that might have an interest in the submitted work. Other relationships: All authors have declared that there are no other relationships or activities that could appear to have influenced the submitted work.

\section{Acknowledgements}


We cordially thank Kazuhiro Nakajima, M.T., Department of Diagnostic Pathology, Shimada City General Medical Center, Shimada, Shizuoka, Japan, and Kohei Watanabe, M.T., Special Reference Laboratories, Hamura, Tokyo, Japan, for their excellent technical assistance and secretarial help. The skillful technical assistance by Naoki Ooishi, M.T., and Kuniaki Muramatsu, M.T., Department of Diagnostic Pathology, Shimada City General Medical Center, Shimada, are also acknowledged. There were no funding sources for reporting the present study.

\section{References}

1. Papadimitriou JC, Drachenberg CB, Brenner DS, Newkirk C, Trump BF, Silverberg SG: "Thanatosomes": a unifying morphogenetic concept for tumor hyaline globules related to apoptosis. Hum Pathol. 2000, 31:1455-65. 10.1053/hupa.2000.20376

2. Dikov DI, Auriault ML, Boivin JF, Sarafian VS, Papadimitriou JC: Hyaline globules (thanatosomes) in gastrointestinal epithelium: pathophysiologic correlations. Am J Clin Pathol. 2007, 127:792-799. 10.1309/URPQC0KYE97WG111

3. Kao GF, Johnson FB, Sulica VI: The nature of hyaline (eosinophilic) globules and vascular slits of Kaposi's sarcoma. Am J Dermatopathol. 1990, 12:256-67. 10.1097/00000372-199006000-00006

4. del Rosario AD, Bui HX, Singh J, Ginsburg R, Ross JS: Intracytoplasmic eosinophilic hyaline globules in cartilaginous neoplasms: a surgical, pathological, ultrastructural, and electron probe $\mathrm{x}$-ray microanalytic study. Hum Pathol. 1994, 25:1283-1289. 10.1016/0046-8177(94)90086-8

5. Abdelzaher E, Elwany A, Amr SA: Malignant peripheral nerve sheath tumour with perineurial differentiation and hyaline eosinophilic globules (thanatosomes): A rare tumour. Malays J Pathol. 2018, 40:355-8.

6. Ma L, Liu YP, Geng CZ, Tian ZH, Wu GX, Wang XL: Undifferentiated embryonal sarcoma of liver in an old female: case report and review of the literature. World J Gastroenterol. 2008, 14:7267-70. 10.3748/wig. 14.7267

7. Dei Tos AP, Mertens F, Pillay N: WHO Classification of Tumors $•$ 5th edition $•$ Soft Tissue and Bone Tumours. WHO Classification of Tumours Editorial Board (ed): IARC Press, Lyon (France); 2020. https://www.iarc.who.int/news-events/publication-of-the-who-classification-of-tumours-5th-editionvolume-3-soft-tissu....

8. Muretto P, Lemma E, Grianti C, Staccioli MP, Fabbretti G, Sbarbati A: Inflammatory malignant fibrous histiocytoma of the kidney: an immunohistochemical and ultrastructural study. Tumori. 1985, 71:147-53.

9. Ohmori T, Arita N, Sano A, et al.: Malignant fibrous histiocytoma showing cytoplasmic hyaline globules and stromal osteoids. A case report with light and electron microscopic, histochemical, and immunohistochemical study. Acta Pathol Jpn. 1986, 36:1931-41. 10.1111/j.1440-1827.1986.tb02258.x

10. Tsutsumi Y: Electron microscopic study using formalin-fixed, paraffin-embedded material, with special reference to observation of microbial organisms and endocrine granules. Acta Histochem Cytochem. 2018, 51:63-71. 10.1267/ahc.18012

11. Tachibana M, Tsutsumi Y: Colitis nucleomigrans: the third type of microscopic colitis (part 2). An ultrastructural study. Pathol Int. 2020, 70:761-6. 10.1111/pin.12995

12. Hartel PH, Jackson J, Ducatman BS, Zhang P: CD99 immunoreactivity in atypical fibroxanthoma and pleomorphic malignant fibrous histiocytoma: a useful diagnostic marker. J Cutan Pathol. 2006, 33 Suppl 2:24-8. 10.1111/j.1600-0560.2006.00492.x

13. Pérot G, Mendiboure J, Brouste V, et al.: Smooth muscle differentiation identifies two classes of poorly differentiated pleomorphic sarcomas with distinct outcome. Mod Pathol. 2014, 27:840-50. 10.1038/modpathol.2013.205

14. Binh MB, Sastre-Garau X, Guillou L, et al.: MDM2 and CDK4 immunostainings are useful adjuncts in diagnosing well-differentiated and dedifferentiated liposarcoma subtypes: a comparative analysis of 559 soft tissue neoplasms with genetic data. Am J Surg Pathol. 2005, 29:1340-7. 10.1097/01.pas.0000170343.09562.39

15. Kim L, Kim YS, Lee JS, et al.: Ciliated muconodular papillary tumor of the lung harboring BRAF V600E mutation and p16INK4a overexpression without proliferative activity may represent an example of oncogene-induced senescence. J Thorac Dis. 2017, 9:E1039-44. 10.21037/jtd.2017.11.120

16. Righi A, Gambarotti M, Sbaraglia M, Sisto A, Ferrari S, Dei Tos AP, Picci P: p16 expression as a prognostic and predictive marker in high-grade localized osteosarcoma of the extremities: an analysis of 357 cases. Hum Pathol. 2016, 58:15-23. 10.1016/j.humpath.2016.07.023

17. Beranek JT: Thanatosomes and cardiomyocyte apoptotic bodies . Hum Pathol. 2001, 32:894-5. 10.1053/hupa.2001.26452

18. Dikov D, Roland J, Chatelet FP, Cywiner-Golenzer C, Dimitrakov J: Hyaline globules (thanatosomes) in prostate disease. Am J Surg Pathol. 2003, 27:700-2. 10.1097/00000478-200305000-00016

19. D'Alfonso TM, Ginter PS, Salvatore SP, Antonio LB, Hoda SA: Phylloides tumor with numerous thanatosomes ("death bodies"): a report of two cases and a study of thanatosomes in breast tumors. Int J Surg Pathol. 2014, 22:337-42. 10.1177/1066896913482728

20. Panicker NK, Buch AC, Patel AR: Breast carcinoma with numerous large "thanatosomes". J Cancer Res Ther. 2015, 11:980-2. 10.4103/0973-1482.157304

21. Gown AM, Willingham MC: Improved detection of apoptotic cells in archival paraffin sections: immunohistochemistry using antibodies to cleaved caspase 3. J Histochem Cytochem. 2002, 50:449-54. 10.1177/002215540205000401

22. Tsutsumi Y, Kamoshida S: Pitfalls and caveats in histochemically demonstrating apoptosis . Acta Histochem Cytochem. 2003, 36:271-284. 10.1267/ahc.36.271

23. Thornberry NA, Rano TA, Peterson EP, et al.: A combinatorial approach defines specificities of members of the caspase family and granzyme B. Functional relationships established for key mediators of apoptosis. I Biol Chem. 1997, 272:17907-11. 10.1074/jbc.272.29.17907

24. Leite AF, Bernardo VG, Buexm LA, Fonseca EC, Silva LE, Barroso DR, Lourenço Sde Q: Immunoexpression of cleaved caspase- 3 shows lower apoptotic area indices in lip carcinomas than in intraoral cancer. J Appl Oral 


\section{Cureus}

Sci. 2016, 24:359-65. 10.1590/1678-775720160156

25. Liu PF, Hu YC, Kang BH, et al.: Expression levels of cleaved caspase-3 and caspase-3 in tumorigenesis and prognosis of oral tongue squamous cell carcinoma. PLoS One. 2017, 12:e0180620.

10.1371/journal.pone.0180620

26. Fang Y, Li J, Wu Y, Gui J, Shen Y: Costunolide Inhibits the Growth of OAW42-a multidrug-resistant human ovarian cancer cells by activating apoptotic and autophagic pathways, production of reactive oxygen species (ROS), cleaved caspase-3 and cleaved caspase-9. Med Sci Monit. 2019, 25:3231-7. 10.12659/MSM.914029

27. Murphy BM, Martin SJ: Caspases: structure, activation pathways, and substrates. Essentials of apoptosis. a guide for basic and clinical research. Yin X, Dong Z (ed): Springer, New York; 2003.

28. Akutsu M, Dikic I, Bremm A: Ubiquitin chain diversity at a glance . J Cell Sci. 2016, 129:875-80. $10.1242 /$ jcs. 183954

29. Grumati P, Dikic I: Ubiquitin signaling and autophagy. J Biol Chem. 2018, 293:5404-13. 10.1074/jbc.TM117.000117

30. Lowe J, Blanchard A, Morrell K, et al.: Ubiquitin is a common factor in intermediate filament inclusion bodies of diverse type in man, including those of Parkinson's disease, Pick's disease, and Alzheimer's disease, as well as Rosenthal fibres in cerebellar astrocytomas, cytoplasmic bodies in muscle, and mallory bodies in alcoholic liver disease. J Pathol. 1988, 155:9-15. 10.1002/path.1711550105

31. Sato S, Kitamura H, Ghazizadeh M, et al.: Occurrence of hyaline droplets in renal biopsy specimens: an ultrastructural study. Med Mol Morphol. 2005, 38:63-71. 10.1007/s00795-004-0272-1

32. Mossuto MF, Ami D, Anelli T, Fagioli C, Doglia SM, Sitia R: Biochemical nature of Russell Bodies . Sci Rep. 2015, 5:12585. 10.1038/srep12585

33. McConnell EE, Talley FA: Intracytoplasmic hyaline globules in the adrenal medulla of laboratory animals . Vet Pathol. 1977, 14:335-40. 10.1177/030098587701400502

34. Tachibana M, Koreyasu R, Kamimura K, Tsutsumi Y: Pancreatic intraductal papillary mucinous neoplasm with hyaline globules (thanatosomes): report of two cases. Intern Med Case Rep J. 2021, 14:1-7. 10.2147/IMCRJ.S309766 